\title{
Trabectome surgery for primary and secondary open angle glaucomas
}

\author{
Jens F. Jordan • Thomas Wecker • Christian van Oterendorp • Alexandra Anton • \\ Thomas Reinhard $\cdot$ Daniel Boehringer $\cdot$ Matthias Neuburger
}

Received: 7 June 2013 /Revised: 20 August 2013 / Accepted: 8 October 2013 / Published online: 26 October 2013

(C) The Author(s) 2013. This article is published with open access at Springerlink.com

\begin{abstract}
Purpose In most forms of open angle glaucoma, the trabecular meshwork is the main barrier for aqueous humor outflow, causing elevated intraocular pressure (IOP). The Trabectome is a minimal invasive device for the surgical treatment of open angle glaucoma, particularly eliminating the juxtacanalicular meshwork. This study was conducted to compare the effectiveness and complication profile among different glaucoma subgroups.

Methods Single center prospective observational study. There were 557 consecutive eyes of 487 patients included in this study. Trabectome surgery was performed either alone or in combination with cataract surgery. Intraoperative and postoperative complications were documented systematically. Main outcome measures were IOP reduction over time and the preoperative and postoperative number of IOP-lowering medications. Due to subgroup sizes, only data from eyes with primary open angle glaucoma and pseudoexfoliation glaucoma were processed for statistical analysis.

Results For the 261 eyes classified as primary open angle glaucoma, preoperative IOP was $24 \pm 5.5 \mathrm{mmHg}$ (mean \pm $\mathrm{SD}$ ) under $2.1 \pm 1.3$ IOP-lowering medications. After a mean follow-up of $204 \pm 238$ days, IOP was reduced to $18 \pm$ $6.1 \mathrm{mmHg}$, and medication was reduced to $1.2 \pm 1.1$. For the 173 eyes classified as pseudoexfoliation glaucoma, after a mean follow-up of $200 \pm 278$ days, IOP was reduced from $25 \pm 5.9 \mathrm{mmHg}$ to $18 \pm 8.2 \mathrm{mmHg}$, and medication was reduced from $2.0 \pm 1.2$ to $1.1 \pm 1.1$. A Cox proportional hazards
\end{abstract}

J. F. Jordan $(\bowtie) \cdot$ T. Wecker $\cdot$ A. Anton $\cdot$ T. Reinhard $\cdot$

D. Boehringer $\cdot M$. Neuburger

University Eye Hospital, Killianstr. 5, 79106 Freiburg, Germany

e-mail: jens.jordan@uniklinik-freiburg.de

C. van Oterendorp

University Eye Hospital, Robert-Koch-Str. 40, 37075 Göttingen,

Germany model hinted forward superiority of the combined surgery cases (Trabectome+Phaco+intraocular lens) in comparison to Trabectome surgery only in phakic or pseudophakic eyes. No serious complications were observed.

Conclusions Minimal invasive glaucoma surgery with the Trabectome seems to be safe and effective. The subgroup analysis of different kinds of open angle glaucomas presented in this study may help in first-line patient selection. The lack of ocular surface alterations makes it a valuable addition to glaucoma surgery.

Keywords Primary and secondary open angle glaucoma . Trabectome $\cdot$ Minimal invasive $\cdot$ Combined surgery

\section{Introduction}

Glaucoma is one of the leading causes for irreversible blindness worldwide [1]. So far, the only proven therapy to slow down the process of optic nerve atrophy is to lower intraocular pressure. Classic glaucoma filtration surgery by means of trabeculectomy or episcleral aqueous drainage implants is the most effective way of lowering intraocular pressure permanently down to low-normal levels. Although both surgical procedures are well established, potentially dangerous intraoperative and postoperative complications might appear in non-negligible frequency. This has led to the development of non-penetrating glaucoma surgery (NPGS) and, more recently, minimal invasive glaucoma surgery. The minimal invasive surgical approach does not alter the conjunctiva, acts in a postoperatively closed and therefore stable eye, offers an excellent opportunity for combined cataract-glaucoma surgery and is further characterized by a minimized intraoperative and postoperative risk profile.

The Trabectome uses a minimal-invasive, gonioscopic clear-cornea surgical approach to reduce outflow resistance 
by removing the juxtacanalicular trabecular meshwork. This is altered pathologically in different forms of glaucoma, acting more as a barrier for the aqueous than as an active and regulative tissue as it is known to be in healthy eyes. Collector channels in the scleral wall of Schlemm's canal are exposed to the anterior chamber and recruited for aqueous drainage into the scleral and episcleral venous plexus.

This prospective, non-comparative study is to report comprehensive follow-up data after Trabectome surgery in different forms of open angle glaucoma.

\section{Methods}

Ethics statement This prospective non-randomized observational study was approved by the local ethics committee (University of Freiburg Ethics Committee No. 235/10_160678) and followed the regulations of GCP and the Declaration of Helsinki. It was designed as an analysis of effectiveness contrary to a controlled efficacy analysis.

After written informed consent, a total of 557 consecutive eyes of 487 Caucasian patients were included from June 2009 to June 2013. In the eyes included, intraocular pressure (IOP) was uncontrolled under topical and/or systemic antiglaucoma medication. Target pressure for these eyes was a reduction in intraocular pressure by at least $20 \%$ from baseline. With the Trabectome, postoperative IOP values below $15 \mathrm{mmHg}$ are unlikely to be achieved. Therefore, absolute target pressures below $15 \mathrm{mmHg}$ were considered as not suitable for inclusion into this study. Patients presenting with a concomitant cataract (visual acuity $\leq 20 / 32$ ) were offered combined surgery (Trabectome + phacoemulsification + intraocular lens).

Surgery was performed by two surgeons (JFJ and MN) using the Trabectome ${ }^{\circledR}$ system, including the Trabectome single-use handpiece including an irrigation-aspiration (I/ A) system (Neomedix Inc., Tustin, USA). In combined surgery, the Trabectome surgery was performed prior to phacoemulsification. After topical anaesthesia using proxymetacainehydrochloride eye drops (Proparakain-POS $0.5 \%{ }^{\circledR}$, Ursapharm, Germany), a $1.7 \mathrm{~mm}$ clear cornea tunnel was made; lidocain $1 \%$ (Xylocain ${ }^{\circledR}$, AstraZeneca, Germany) was instilled into the anterior chamber for intraocular anesthesia.

A methylcellulose-based viscoelastic (Ocucoat ${ }^{\circledR}$, Bausch \& Lomb) was used in all cases to stabilize the anterior chamber during surgery as recommended by the manufacturer of the Trabectome. Surgery was performed under gonioscopic control using the modified Swan-Jacob-Lens. The trabecular meshwork was removed over 90 to $120^{\circ}$ starting with a power of $0.8 \mathrm{~W}$, increasing in steps of 0.1 until the electrosurgical effect allowed smooth removal of the juxtacanalicular tissue without tearing. Viscoelastic was removed using the provided I/A-system with a modified Simco-cannula.
Pilocarpine $2 \%$ eye drops, a topical steroid and antibiotic ointment were instilled after surgery. All eyes were given Pilocarpine $2 \%$ tid for the first week after surgery, and bid thereafter for four more weeks. Steroid eyedrops (prednisolone $1 \%$ ) were given five times daily or more frequently in the case of stronger postoperative intraocular reflux bleeding during the first week after surgery. Steroids were tapered over four weeks after surgery. Intraocular pressure (IOP)-lowering medication was given topically or systemically according to individual postoperative IOP-levels.

Intraocular pressure was recorded in a masked fashion using Goldmann applanation tonometry. Values were recorded by an independent person. IOP measurements were recorded on postoperative day one, after one week, one month, and every three months thereafter.

Absolute success was defined as a reduction of IOP from baseline of at least $20 \%$ without the need of additional medication. Qualified success was a reduction of IOP from baseline of at least $20 \%$ with or without the need for topical antiglaucomatous medication. Data was collected using an intranet-based relational database.

To account for censoring, we estimated the cumulative percentages of absolute and qualified success as well as the failure rates over time with the Kaplan-Meier method. Differences between subgroups were tested with the logrank statistics. Statistical significance was assumed for $p \leq 0.05$. We fitted a Cox proportional hazards model to assess the risk factors for failure or subsequent glaucoma surgery in that eye. We, i.e., tested whether cataract surgery at time of Trabectome surgery is beneficial contrary to performing Trabectome in pseuophacic eyes against the background of age at time of surgery and glaucoma entities.

\section{Results}

A total of 557 eyes of 487 patients have been included in this comprehensive effectiveness analysis. Please see Table 1 for the detailed demographic data and descriptive statistics on all patients. Due to the small sample size of most glaucoma entities, further subgroup analysis was only performed for the eyes classified as Primary Open Angle Glaucoma (POAG, $n=261$ ) and the eyes classified as Pseudoexfoliation Glaucoma (XFG, $n=173$ ).

Figure 1 shows the Kaplan-Meier cumulative survival analysis, displaying absolute success (Fig. 1a; $\Delta$ IOP $\geq-20 \%$ from baseline, no medication) and qualified success (Fig. 1b; $\Delta \mathrm{IOP} \geq-20 \%$ from baseline, topical medication allowed) over time. For qualified success, the difference between the two groups proved to be statistically significant at $p=0.01$.

Intraocular pressure (IOP) and number of topical medications (qualified success) over the follow-up period 


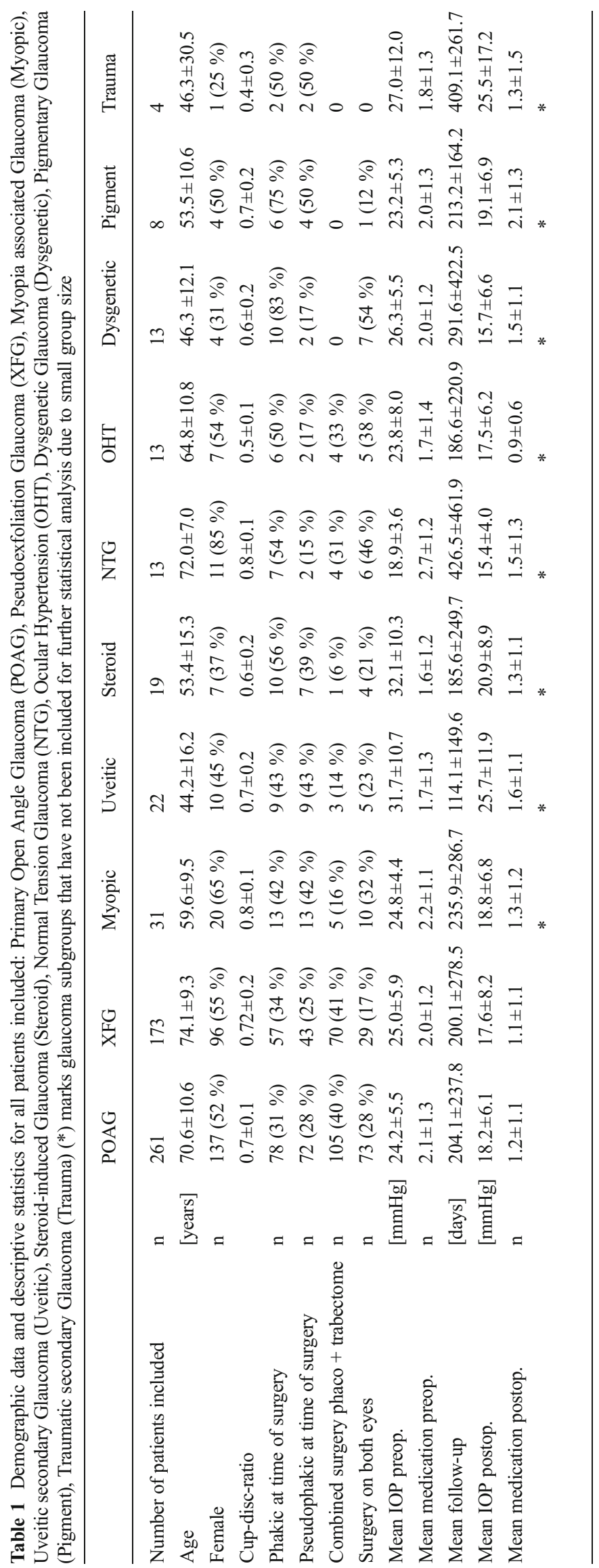


Fig. 1 a Absolute success: Kaplan-Meier cumulative survival of all eyes classified as Primary Open Angle Glaucoma (POAG) or Pseudoexfoliation Glaucoma (XFG) over time, absolute success $(\triangle \mathrm{IOP} \geq-20 \%$ from baseline, no additional IOP lowering medication). Please note the steep drop of the KaplanMeier curve for both, POAG and $\mathrm{XFG}$, resembling the persistent need for topical medication after Trabectome surgery in most eyes. b Qualified success: KaplanMeier cumulative survival of all eyes classified as Primary Open Angle Glaucoma (POAG) or Pseudoexfoliation Glaucoma (XFG) over time, qualified success $((\Delta \mathrm{IOP} \geq-20 \%$ from baseline, topical medication allowed). The difference between the two groups was significant at $p=0.01$

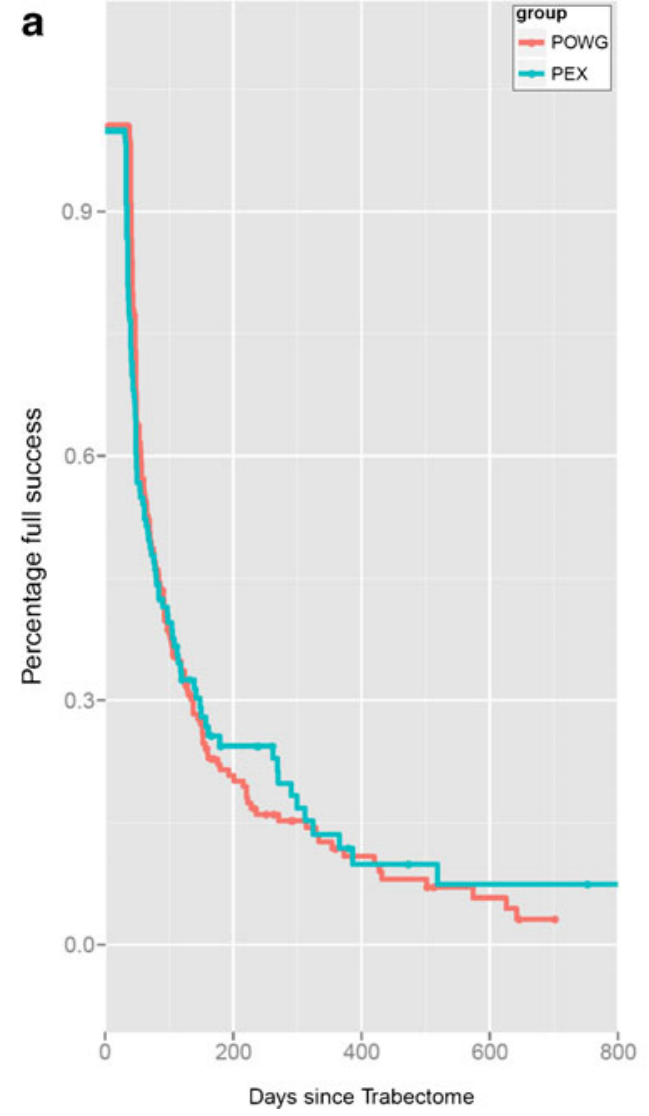

are displayed for POAG (Fig. 2) and for XFG (Fig. 3). Groups are sub-classified as phakic or pseudophakic at the time of Trabectome surgery, or having undergone combined Trabectome - cataract (Trabectome + phaco + intraocular lens) surgery.

We further analyzed the effectiveness after Trabectomeonly surgery in phakic and pseudophakic eyes, as well as the effectiveness of the combined surgery (Trabectome + Phacoemulsification + intraocular lens implantation). A Cox proportional hazards model (corrected for age and lens state) hints towards superiority of the combined surgery over the Trabectome-only surgery when performed in either phakic or pseudophakic eyes (Table 2).

No severe intraoperative and postoperative complications were observed. Minor events included reflux bleeding that occurred in $92 \%$ of patients and resolved spontaneously. Only two patients needed one lavage of the anterior chamber to remove the blood. This was not associated with failure. However, there was one case of intraoperative ciliolenticular block that occurred during anterior chamber lavage with acetylcholine (Miochol-E ${ }^{\circledR}$, Bausch \& Lomb). It resolved under postoperative topical cycloplegic treatment. No postoperative hypotony (defined as an $\mathrm{IOP}<6 \mathrm{mmHg}$ ) was observed. Eight percent of patients presented with an acute postoperative rise in IOP $(>30 \mathrm{mmHg}$ ) that was controlled with temporary systemic carboanhydrase inhibitors (CAI). Three patients developed

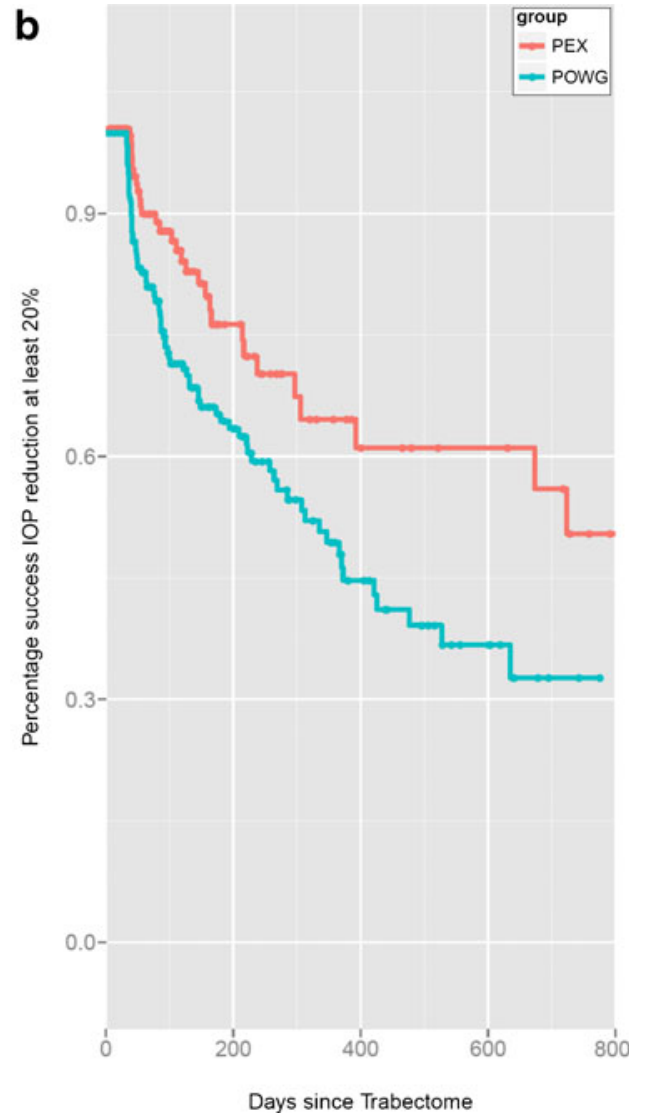

clinically evident cystoid macular edema after combined surgery that resolved under topical anti-inflammatory treatment (prednisolone and diclofenac eye-drops) and systemic carboanhydrase inhibitor. IOP values measured under systemic CAI therapy were not collected for statistical analysis.

For the patients of failed Trabectome surgery, a subsequent trabeculectomy with Mitomycin C was performed with a formerly untouched conjunctiva due to uncontrolled IOP. In two patients, a second Trabectome approach was performed but did not result in any further IOP reduction. In both cases, this was followed by a trabeculectomy.

\section{Discussion}

In this prospective, noncomparative study, the Trabectome seems to be a safe method of minimal invasive glaucoma surgery. Our data also hint towards the effectiveness in reducing IOP and postoperative number of medications.

In the 261 consecutive eyes classified as primary open angle glaucoma, we reached an overall IOP reduction of $25 \%$, with a simultaneous reduction in antiglaucoma medication of $43 \%$. For the 173 eyes classified as pseudoexfoliation glaucoma, we found an overall IOP reduction of $30 \%$ with a simultaneous reduction in medication of $44 \%$. The Cox proportional hazards model correcting for age and lens state hinted towards 
a

POAG

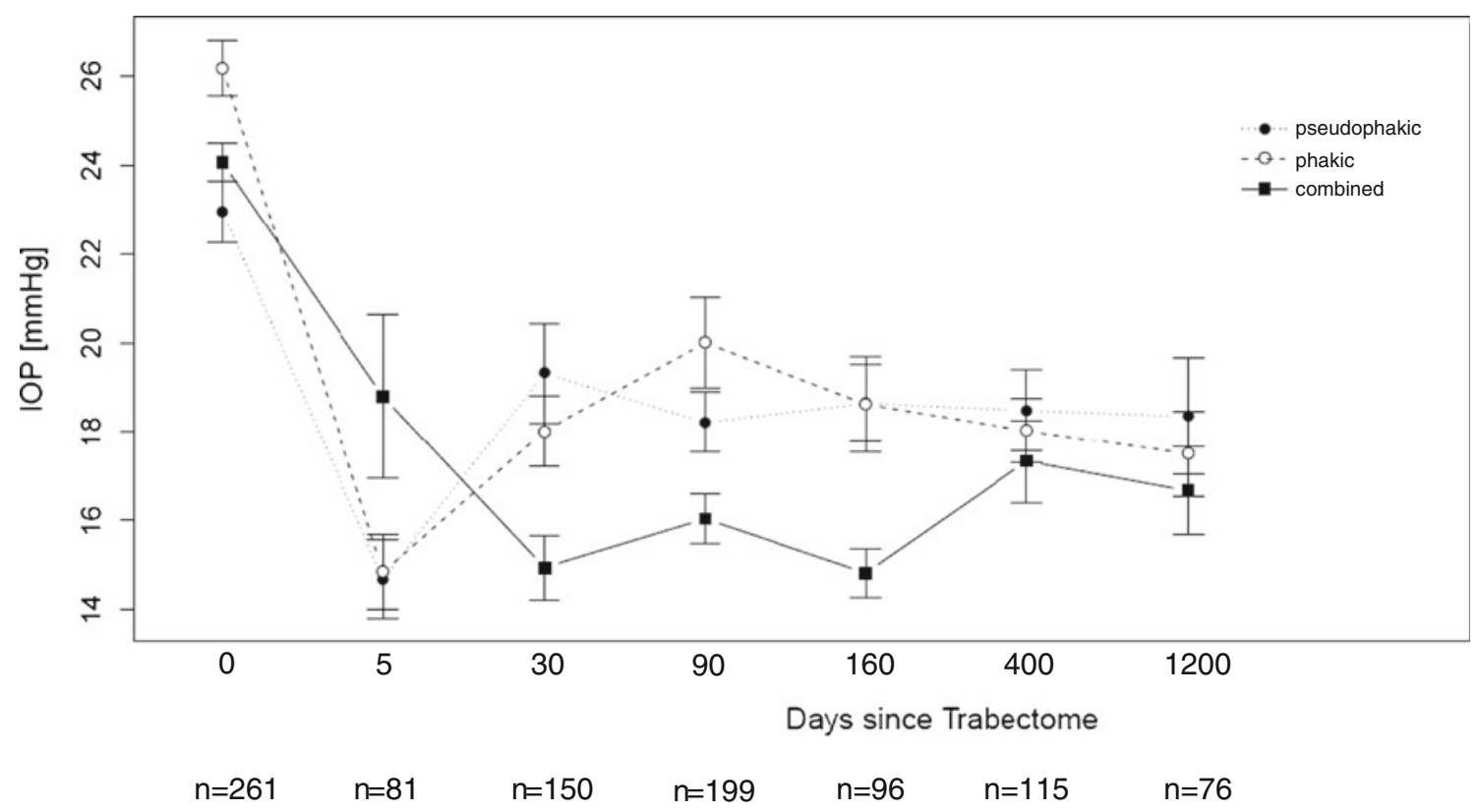

b

POAG

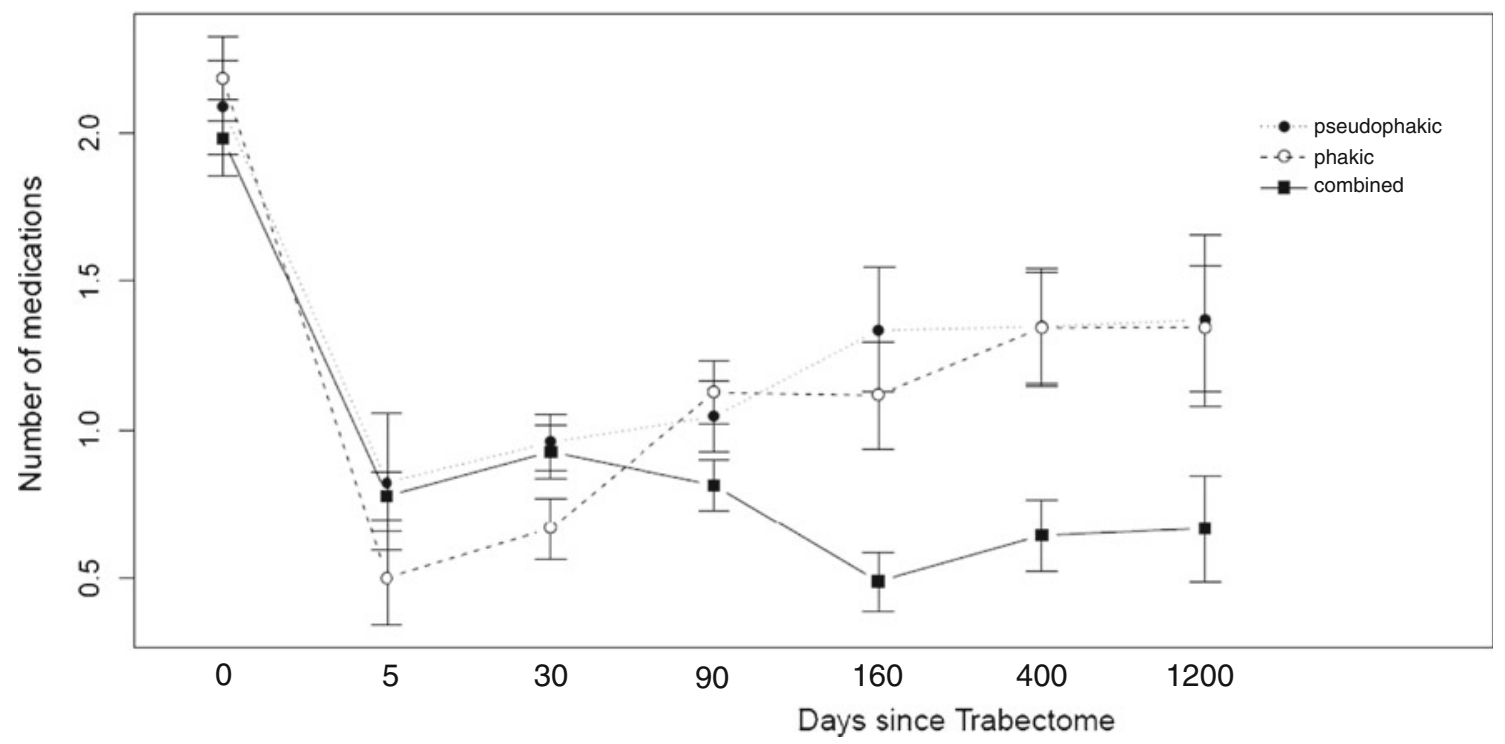

Fig. 2 a Postoperative IOP data (ordinate $[\mathrm{mmHg}]$ ) over time (abscissa [days after surgery]) from eyes classified as POAG, given separately for the eyes being phakic or pseudophakic at the time of Trabectome surgery, and for the eyes having undergone combined cataract and Trabectome surgery. Time ' 0 ' gives the preoperative IOP value. $\mathrm{N}$ (below abscissa) gives the number of patients included at each time point. b Qualified

superiority of the combined surgery cases (Trabectome + Phaco + IOL), with a trend towards younger age being a risk factor for subsequent glaucoma surgery.

In the literature, the largest published data pool consists of 1127 eyes, a mixed population of different subgroups of open angle glaucomas [2]. These data were collected and success: Number of postoperative topical medications needed (ordinate [n]) over time (abscissa [days after surgery]) from eyes classified as POAG, given separately for the eyes being phakic or pseudophakic at the time of Trabectome surgery, and for the eyes having undergone combined cataract and Trabectome surgery. Time ' 0 ' gives the preoperative number of topical medications

published in affiliation with Neomedix Inc. In 738 of these eyes, only Trabectome surgery (without cataract extraction) was performed. After two years of observation, the IOP was reduced by $40 \%(n=46)$, medication could be reduced by $60 \%$. In 366 of the eyes, Trabectome surgery was performed in combination with cataract surgery. Starting 
a XFG

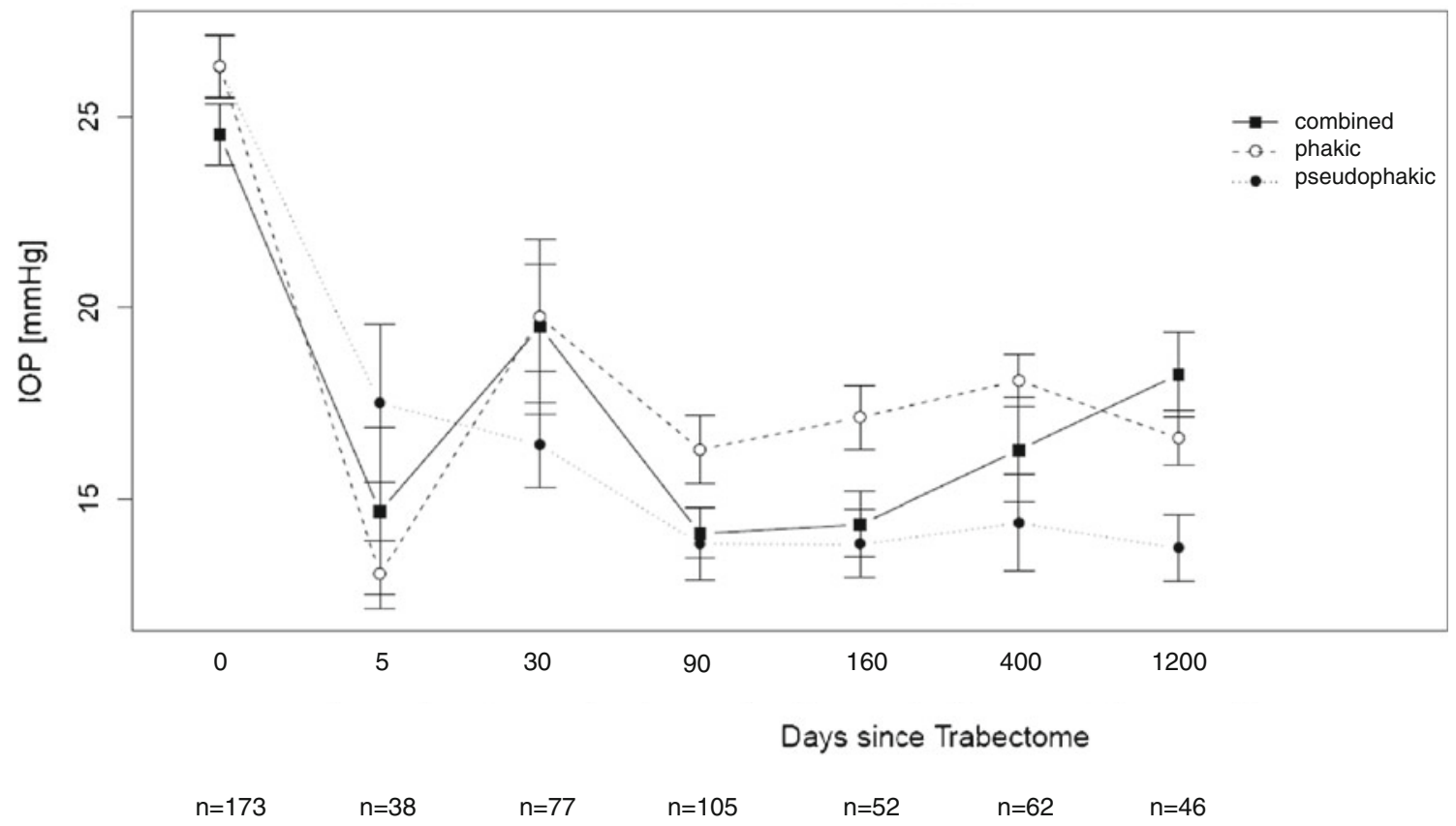

b $\quad$ XFG

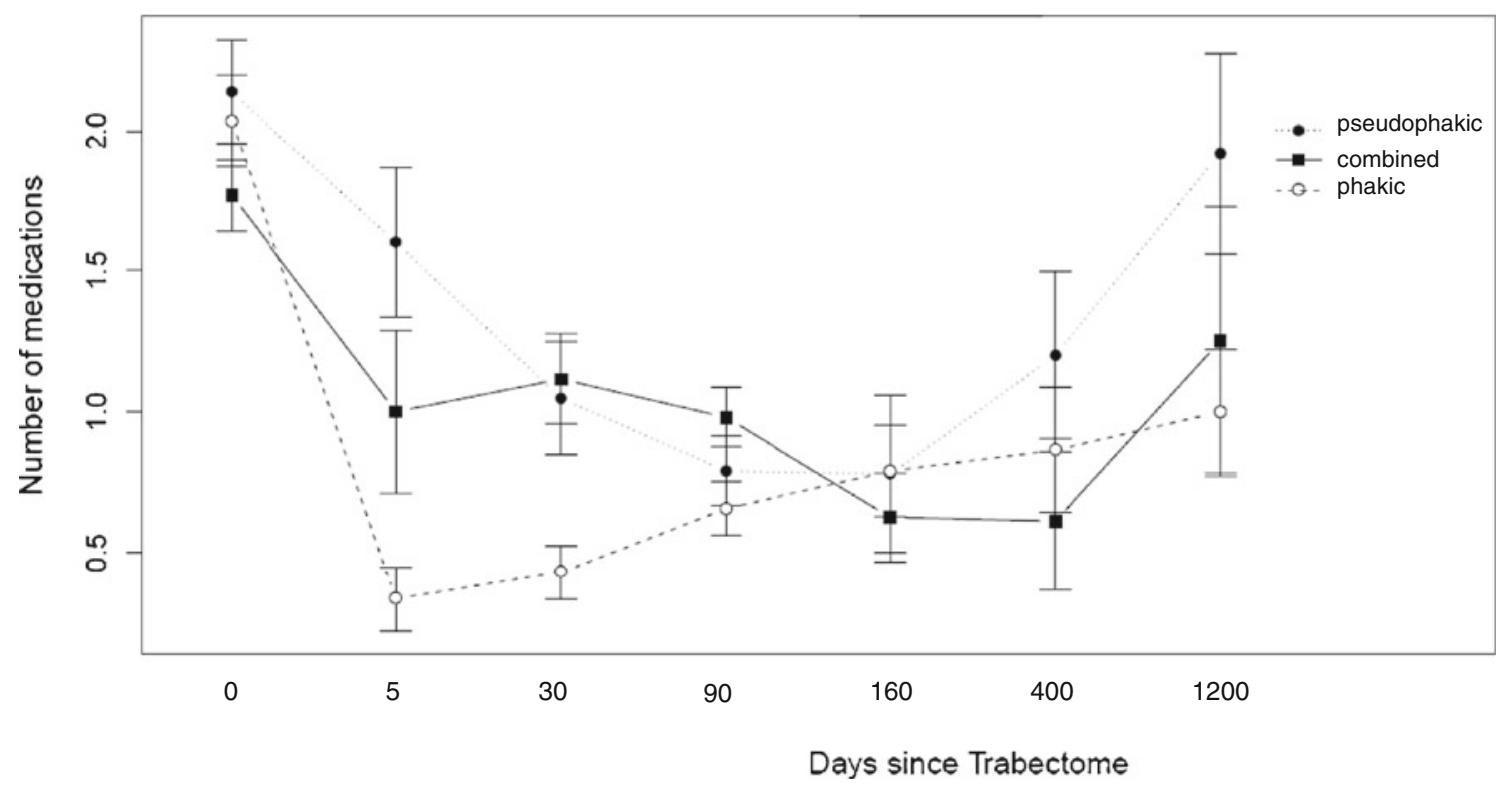

Fig. 3 a Postoperative IOP data (ordinate $[\mathrm{mmHg}]$ ) over time (abscissa [days after surgery]) from eyes classified as XFG, given separately for the eyes being phakic or pseudophakic at the time of Trabectome surgery, and for the eyes having undergone combined cataract and Trabectome surgery. Time ' 0 ' gives the preoperative IOP value. $\mathrm{N}$ (below abscissa) gives the number of patients included at each time point. b Qualified

success: Number of postoperative topical medications needed (ordinate [n]) over time (abscissa [days after surgery]) from eyes classified as XFG, given separately for the eyes being phakic or pseudophakic at the time of Trabectome surgery, and for the eyes having undergone combined cataract and Trabectome surgery. Time ' 0 ' gives the preoperative number of topical medications

with a lower baseline IOP, it was reduced by $20 \%$ with a concomitant reduction in medication by $57 \%$. Also, $78 \%$ of all patients had intraoperative reflux bleeding from the collector channels. During the follow-up period, $5.9 \%$ of patients had to be converted to trabeculectomy, and $1.6 \%$ needed glaucoma implant surgery due to uncontrolled IOP. In this data report, no subgrouping of the different forms of glaucoma was provided. 
Table 2 Cox proportional hazards model for surgical failure (POAG and $\mathrm{XFG).} \mathrm{Reference} \mathrm{is} \mathrm{pseudophacic} \mathrm{lens} \mathrm{state} \mathrm{at} \mathrm{time} \mathrm{of} \mathrm{surgery.} \mathrm{Combined}$ surgery (Trabectome + Cataract) significantly lowers the risk for surgical failure in comparison to pseudophakic patients. There is a trend towards increasing age being beneficial for a successful surgical outcome. HR Hazard Ratio, CI - Confidence Interval, phakic - phakic at time of Trabectome surgery, combined - Trabectome + phaco, age - age at time of surgery. Statistical significance was assumed for $p<0.05$

\begin{tabular}{lllll}
\hline & HR & CI lower 95 & CI upper 95 & $\mathrm{p}$ \\
\hline Phakic & 1.12 & 0.54 & 2.29 & 0.759 \\
Combined & 0.20 & 0.07 & 0.62 & 0.005 \\
Age & 0.98 & 0.96 & 1.01 & 0.242 \\
\hline
\end{tabular}

Yet another study with comparable preoperative IOPvalues reports on the 1-year success rate after Trabectome surgery alone and after combined surgery. In the Trabectome group $(n=538)$, intraocular pressure was reduced by $31 \%$ $(26.3 \pm 7.7 \mathrm{mmHg} \rightarrow 16.6 \pm 4 \mathrm{mmHg})$, the concomitant medications by $28 \%(n=2.9 \pm 1.3 \rightarrow n=2.1 \pm 1.4)$. In the combined surgery group $(n=290)$, the reduction in IOP was only $23 \%$ (to $15.6 \pm 3.7 \mathrm{mmHg}$ ), with a preoperative baseline of $20.2 \pm 6 \mathrm{mmHg}$. Medication was reduced by $33 \%(n=2.5 \pm$ $1.1 \rightarrow n=1.7 \pm 1.3) .65 \%$ of the Trabectome group and $87 \%$ of the phaco-Trabectome group met the criterion of qualified [3].

Ting et al., compared the outcomes of eyes classified as POAG or XFG, either after Trabectome surgery alone or after combined surgery by a retrospective, central database analysis[4]. For POAG, 450 Trabectome only and 263 combined surgery cases were included. After one year, IOP was reduced from $25.5 \pm 8 \mathrm{mmHg}$ to $16.8 \pm 4 \mathrm{mmHg}(-34 \%)$ and from $19.9 \pm 5 \mathrm{mmHg}$ to $15.6 \pm 3 \mathrm{mmHg}(-22 \%)$, medication was reduced by $18.5 \%$ and $29 \%$, respectively. For XFG, in the 67 Trabectome only eyes, IOP was reduced by $45 \%$ from $29.0 \pm 8 \mathrm{mmHg}$ to $16.1 \pm 4 \mathrm{mmHg}$, in the 45 combined cases by $35 \%$ from $21.7 \pm 8 \mathrm{mmHg}$ to $14.2 \pm$ $3 \mathrm{mmHg}$ after one year. Medication was reduced by $29 \%$ and $36 \%$, respectively.

The published results are in agreement with the results from our study in terms of overall reduction in IOP and medications ( $25 \%$ and $43 \%$ for POAG and $30 \%$ and $44 \%$ for XFG, respectively). In a Cox proportional hazards model correcting for age and lens-state, in our study group there was a significant superiority in terms of sparing further glaucoma surgery for the combined Trabectome + phaco surgery in comparison with Trabectome surgery alone in eyes being either phakic or pseudophakic at the time of operation. The better IOP-lowering effect might in part be caused by the phacoemulsification in its own right. This could bias our finding. However, the Cox proportional hazards model took the pseudophakic state as the reference level. This rules out the intraocular lens as confounder.
Of all eyes that failed, 56 eyes of 54 patients underwent mitomycin-C augmented filtration surgery, i.e., trabeculectomy. It is reported in the literature, that filtration surgery after Trabectome surgery is not affected in its surgical outcome and success rates [5]. We also did not see a higher complication or failure rate than that reported in the literature for primary mitomycin-C augmented trabeculectomy [6-8].

As Trabectome surgery does not alter the ocular surface (this in contrast to nonpenetrating glaucoma surgery, filtration surgery or drainage implants), it is of particular interest for all patients suffering from immunologic ocular surface diseases or having undergone penetrating keratoplasty. Further, it is known from the literature that over $80 \%$ of glaucoma patients suffer from severe ocular surface disturbances due to chronic application of several antiglaucoma drops [9-11]. Early, minimal invasive surgery might be a good opportunity to ease and improve the therapy for those patients $[12,13]$.

Glaucoma surgery of the juxtacanalicular trabecular meshwork aims to overcome the outflow resistance of a pathologically altered tissue[14]. Morphological changes of extracellular matrix and trabecular cells have been well characterized [15-18]. With the Trabectome, the scleral wall of Schlemm's canal with its collector channel openings is exposed to direct aqueous flow. The theory that the main outflow resistance lies in the inner wall of Schlemm's canal would suggest an inevitable, significant IOP reduction after surgery. Yet, all clinical data show limited success rates in almost all forms of open angle glaucoma. It is of particular interest that patients with a widely opened Schlemm's canal on gonioscopy and OCT can present with both target as well as high IOP levels. The introduction of high-resolution imaging techniques has led to new insights into the anatomical structures beyond Schlemm's canal. Applying both Micro-CT and spectral-domain OCT on human eyes, they are able to reveal highly complex patterns of collector channels as well as very irregular intracleral-and episcleral outflow vessels [19-21]. Further experimental studies will be essential to better understand the transport and resorption of aqueous humor across the collector channels and the complex downstream draining and vascular system. This knowledge will be crucial to better understand and predict success and failure of angle surgery with the Trabectome.

Due to the study design as a prospective observational study with the focus on the effectiveness of the Trabectome in different forms of open angle glaucomas, there was no randomization and no control population included. One limitation of this study is the inclusion of both eyes of several individuals ( $n=70$ among all subgroups). Nevertheless, their absolute numbers among the POAG and XFG groups are too small to cause statistically significant interference. Further, the limited numbers in many of the investigated subgroups might be a limiting factor of this study. Therefore, these subgroups have been excluded from statistical analysis. However, in 
order to prevent publication bias, evaluating a relational database requires to at least descriptively reporting all data available. More data is required to further validate our first results and trends.

The results of our study confirm that minimal invasive glaucoma surgery with the Trabectome offers a valuable addition to the armamentarium of glaucoma surgery. The confirmation is convincing because of the facts of a fast operation in topical anesthesia, the low risk and complication profile, and the remaining option for filtration surgery, if necessary. The achievable target pressure makes it a good option for early surgery in eyes with moderate optic disc damage. It might also be considered to reduce topical antiglaucomatous therapy. Consequently, it should not be seen as an alternative to filtration surgery in advanced cases, neither regarding the achievable target pressure nor the definition of surgical success. It proved to be a good option for the combined cataractglaucoma surgery.

Financial disclosure None of the authors has any proprietary or financial interest.

Open Access This article is distributed under the terms of the Creative Commons Attribution License which permits any use, distribution, and reproduction in any medium, provided the original author(s) and the source are credited.

\section{References}

1. Quigley HA, Broman AT (2006) The number of people with glaucoma worldwide in 2010 and 2020. Br J Ophthalmol 90:262-267

2. Minckler D, Mosaed S, Dustin L, Ms BF (2008) Trabectome (trabeculectomy-internal approach): additional experience and extended follow-up. Trans Am Ophthalmol Soc 106:149-159, discussion 159-160

3. Mosaed S, Rhee DJ, Filippopoulos T, Tseng H, Deokule S, Weinreb RN (2010) Trabectome outcomes in adult open-angle glaucoma patients: One-year follow-up. Clin Surg Ophthalmol 28:5-9

4. Ting JLM, Damji KF, Stiles MC (2012) Ab interno trabeculectomy: Outcomes in exfoliation versus primary open-angle glaucoma. J Cataract Refract Surg 38:315-323

5. Jea SY, Mosaed S, Vold SD, Rhee DJ (2012) Effect of a failed trabectome on subsequent trabeculectomy. J Glaucoma 21:71-75
6. Landers J, Martin K, Sarkies N, Bourne R, Watson P (2012) A twenty-year follow-up study of trabeculectomy: risk factors and outcomes. Ophthalmology 119:694-702

7. Gedde SJ, Herndon LW, Brandt JD, Budenz DL, Feuer WJ, Schiffman JC, Tube Versus Trabeculectomy Study Group (2012) Postoperative complications in the Tube Versus Trabeculectomy (TVT) study during five years of follow-up. Am J Ophthalmol 153: 804-814

8. Gedde SJ, Schiffman JC, Feuer WJ, Herndon LW, Brandt JD, Budenz DL, Tube versus Trabeculectomy Study Group (2012) Treatment outcomes in the Tube Versus Trabeculectomy (TVT) study after five years of follow-up. Am J Ophthalmol 153:789-803

9. Van Went C, Brasnu E, Hamard P, Baudouin C, Labbé A (2011) The influence of ocular surface diseases in the management of glaucoma. J Fr Ophtalmol 34:230-237

10. Fechtner RD, Godfrey DG, Budenz D, Stewart JA, Stewart WC, Jasek MC (2010) Prevalence of ocular surface complaints in patients with glaucoma using topical intraocular pressure- lowering medications. Cornea 29:618-621

11. Leung EW, Medeiros FA, Weinreb RN (2008) Prevalence of ocular surface disease in glaucoma patients. J Glaucoma 17:350-355

12. Dietlein TS, Jordan JF, Lüke C, Schild A, Dinslage S, Krieglstein GK (2008) Self-application of single-use eyedrop containers in an elderly population: comparisons with standard eyedrop bottle and with younger patients. Acta Ophthalmol 86:856-859

13. Dietlein TS (2006) Glaucoma in the aged-barriers to understanding and compliance. Ophthalmologe 103:755-758

14. Zhou J, Smedley GT (2005) A trabecular bypass flow hypothesis. J Glaucoma 14:74-83

15. Lütjen-Drecoll E (2005) Morphological changes in glaucomatous eyes and the role of TGFbeta2 for the pathogenesis of the disease. Exp Eye Res 81:1-4

16. Tektas O-Y, Lütjen-Drecoll E (2009) Structural changes of the trabecular meshwork in different kinds of glaucoma. Exp Eye Res 88:769-775

17. WuDunn D (2009) Mechanobiology of trabecular meshwork cells. Exp Eye Res 88:718-723

18. Tamm ER (2009) The trabecular meshwork outflow pathways: Structural and functional aspects. Exp Eye Res 88:648-655

19. Hann CR, Bentley MD, Vercnocke A, Ritman EL, Fautsch MP (2011) Imaging the aqueous humor outflow pathway in human eyes by three-dimensional micro-computed tomography (3D micro-CT). Exp Eye Res 92:104-111

20. Kagemann L, Wollstein G, Ishikawa H, Sigal IA, Folio LS, Xu J, Gong H, Schuman JS (2011) 3D visualization of aqueous humor outflow structures in-situ in humans. Exp Eye Res 93: 308-315

21. Kagemann L, Wollstein G, Ishikawa H, Bilonick RA, Brennen PM, Folio LS, Gabriele ML, Schuman JS (2010) Identification and assessment of Schlemm's canal by spectral-domain optical coherence tomography. Invest Ophthalmol Vis Sci 51:4054-4059 